\title{
Development of Miniaturized Thin-Film Magnetic Field Probes for On-Chip Measurement
}

\author{
N. Ando, N. Masuda, N. Tamaki, T. Kuriyama ${ }^{*}$, M. Saito ${ }^{* *}$, S. Saito ${ }^{* * *}$, K. Kato ${ }^{* *}$, K. Ohashi ${ }^{* * * *}$, and \\ M. Yamaguchi ${ }^{* * * *}$ \\ Jisso and Production Technologies Research Laboratories, NEC Corp., 1120, Shimokuzawa, Sagamihara, Kanagawa 229-1198, Japan \\ * School of Biology-Oriented Science and Technology, Kinki Univ., 930, Nishi-Mitani, Kinokawa, Wakayama 649-6493, Japan \\ ${ }^{* *}$ Nano Technology Research Laboratory, Waseda Univ., 513, Waseda-Tsurumaki-Cho, Shinjuku-ku, Tokyo 162-0041, Japan \\ ${ }^{* * *}$ System Devices Research Laboratories, NEC Corp., 1120, Shimokuzawa, Sagamihara, Kanagawa 229-1198, Japan \\ ${ }^{* * * *}$ Fundamental and Environmental Research Laboratories, NEC Corp., 34 Miyukigaoka, Tsukuba, Ibaraki 305-1111, Japan \\ ${ }^{* * * * *}$ Graduate School of Engineering, Tohoku Univ., 6-6-05 Aramaki-Aza-Aoba, Aoba-ku, Sendai 980-8579, Japan
}

We developed thin-film magnetic field probes with a high spatial resolution aiming to obtain the absolute value of a high-frequency power current on an LSI chip. The spatial resolution was enhanced by miniaturizing the shielded loop coil, which is the detection part of the probe. The minimum outer size of the new coil is $50 \times 22 \mu \mathrm{m}$. In taking measurements with the new probe over a $60-\mu \mathrm{m}$-wide microstrip line used as a device under test (DUT), we found that the probe output decreases by $6 \mathrm{~dB}$ at a distance of $40 \mu \mathrm{m}$. This value is less than half that of our previous probe, which was around $100 \mu \mathrm{m}$. In taking measurements with the new probe over 5 - $\mu \mathrm{m}$-wide microstrip lines used as a DUT, we found that the new probe achieved $10-\mu \mathrm{m}$-class high spatial resolution. This value is comparable to the typical width of global power lines on an LSI chip, which ranges from $10 \mu \mathrm{m}$ to $100 \mu \mathrm{m}$. We estimated the configuration of the lines on an LSI chip that would enable the new probe to achieve a spatial resolution high enough to obtain the absolute value of a high-frequency power current on an LSI chip.

Key words: magnetic near-field measurement, shielded loop coil, miniaturization, spatial resolution, microstrip line

\section{Introduction}

LSI operation generates high-frequency current flowing through power supply lines. This current is considered to be one of the major sources of electromagnetic interference (EMI) ${ }^{1)}$, and could also cause degradation of power integrity (PI) or signal integrity (SI) ${ }^{1)}$. Thus, it is important to monitor this high-frequency power current.

Magnetic near-field measurement can be utilized for contactless current measurement. If the high-frequency current flowing through each power line on an LSI could be obtained from magnetic near-field measurement along with calibration, the obtained data could be effectively used to improve LSI chip design. To obtain the current value, however, magnetic field probes need to have much higher spatial resolution in comparison with the typical width of global power lines on an LSI chip, which ranges from $10 \mu \mathrm{m}$ to $100 \mu \mathrm{m}$. Several studies have been conducted focusing on this issue ${ }^{2-5)}$.

A shielded loop coil ${ }^{6}$ we proposed before is a good representative of a magnetic field probe ${ }^{3-5)}$. We fabricated some magnetic field probes with a shielded loop coil, the detection part of the probe. This coil shows linearity and a wide frequency bandwidth. It also enables time domain measurement. With our previous magnetic field probes we successfully measured the current value flowing through lines on PCBs. However, they had insufficient spatial resolution in comparison with the width of power lines and the line space on LSI chips. In this study, we have tried to develop a new magnetic field probe that enables contactless current measurement at the LSI chip level ${ }^{7)}$. To achieve higher spatial resolution, the shielded loop coil was miniaturized.

\section{Structure of a shielded loop coil}

We previously fabricated various shielded loop coils. One of these coils that was embedded in a multilayer ceramic substrate had an outer size of $1600 \times 800 \mu \mathrm{m}$, while a coil on a glass substrate had an outer size of $680 \mathrm{x}$ $680 \mu \mathrm{m}^{3-5)}$. The shielded loop coil was miniaturized to improve spatial resolution. Figure 1 is a schematic drawing of a shielded loop coil, which is the detection part of the magnetic field probe. The coil of our new probe was fabricated on a glass substrate by using a thin-film process called fine pattern-plating ${ }^{8)}$. In the lithographic process, reduction projection printing using an i-line stepper was used instead of the contact printing used to fabricate our previous coil on a glass substrate. This is because the former technique can pattern under-1- $\mu \mathrm{m}$-wide lines, while the latter one can make only around $5-\mu \mathrm{m}$-wide lines at best. In the new probe, copper and photoresist were used as the conductors and insulators, respectively. Figure 2 is a micrograph of a 


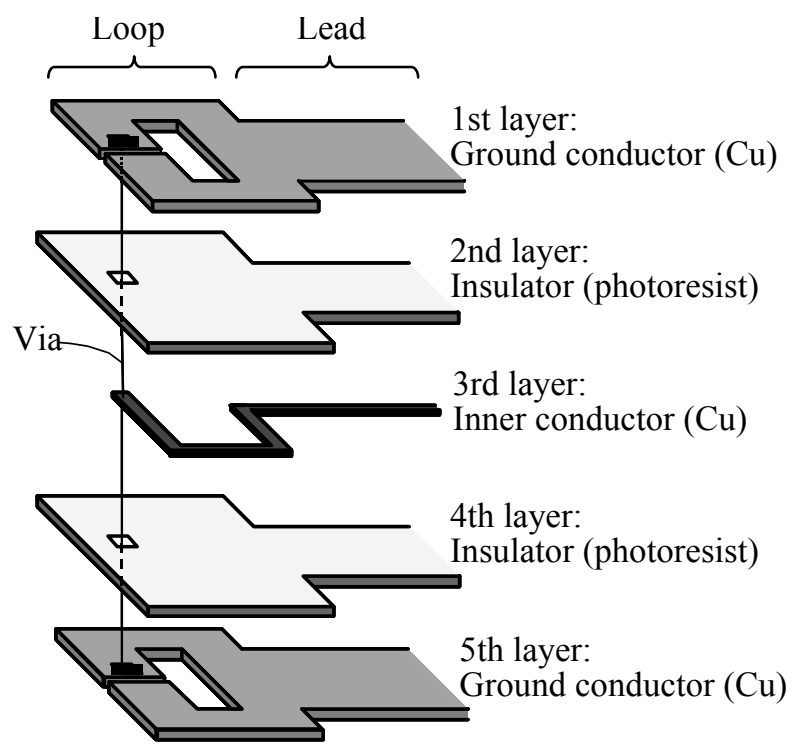

Fig. 1 Schematic of shielded loop coil. Copper and photoresist were used as the conductors and insulators.

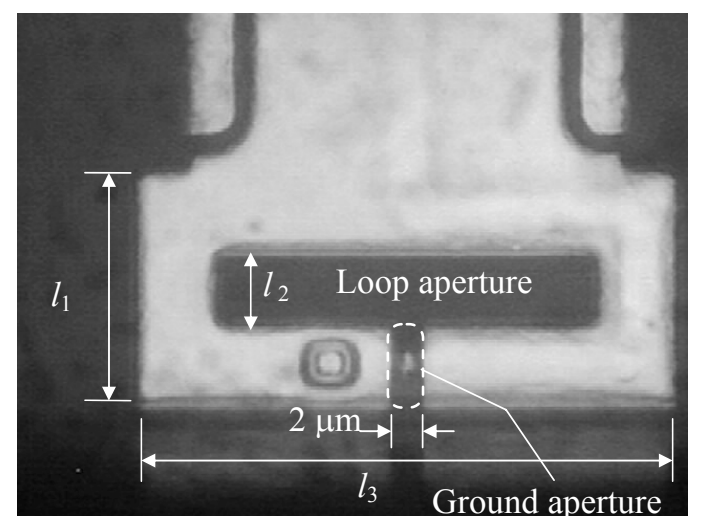

Fig. 2 Micrograph of a shielded loop coil. Ground aperture width is reduced to $2 \mu \mathrm{m}$.

Table 1 Dimensions of our new and previous coils.

\begin{tabular}{|c|c|c|c|}
\hline & $\begin{array}{c}\text { Previous } \\
\text { ceramic coil }\end{array}$ & $\begin{array}{c}\text { Previous } \\
\text { glass coil }\end{array}$ & New coil \\
\hline$l_{1}$ & $800 \mu \mathrm{m}$ & $680 \mu \mathrm{m}$ & $22 \mu \mathrm{m}$ \\
\hline$l_{2}$ & $200 \mu \mathrm{m}$ & $600 \mu \mathrm{m}$ & $6 \mu \mathrm{m}$ \\
\hline
\end{tabular}

shielded loop coil. In Fig. 2, the dimensions $l_{1}$ and $l_{2}$, which represent the respective longitudinal lengths of the coil and the loop aperture, determine the coil's spatial resolution. In our new probe, $l_{1}$ and $l_{2}$ were miniaturized to 22 and $6 \mu \mathrm{m}$, respectively, compared with 680 and 600 $\mu \mathrm{m}$ for our previous probe with a glass substrate (previous glass probe). Table 1 compares the dimensions of our new and previous coils. The dimension $l_{3}$ represents the width of the coil. In the smallest coil, $l_{3}=50 \mu \mathrm{m}$. Considering that the straight section of each global power line on an LSI is usually much longer than the minimum dimension of $l_{3}(50 \mu \mathrm{m})$, the current value can be practically obtained from the measured magnetic field over the LSI. The outer ground conductors have a narrow gap, called the ground aperture, at the edge of the loop coil. The width of the ground aperture was reduced to $2 \mu \mathrm{m}$ by using this finer fabrication process. The distance from the edge of the coil to the edge of the substrate was also reduced to $1 \mu \mathrm{m}$ by grinding the edge surface of the substrate so that the coil can be placed close to a device under test (DUT).

\section{Characteristics of the new probe}

\subsection{Measurement setup}

In a basic evaluation of our new probe, microstrip lines were used as DUTs. Figures 3(a) and (b) show the angled and cross-sectional views of the measurement setup. Measurements were taken above the microstrip lines. The coordinate system was defined as shown in Fig. 3, where the origin of the $x$-axis was at the center of the strip conductor. In Fig. 3(b) the dimensions $h, w$, and $t$ represent the thickness of the dielectric and the width and thickness of the strip conductor, while $\varepsilon_{\mathrm{r}}$ represents the

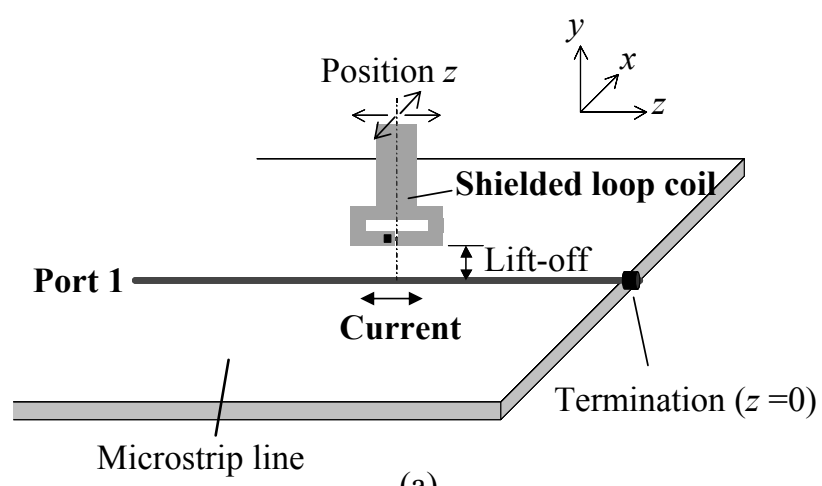

(a)

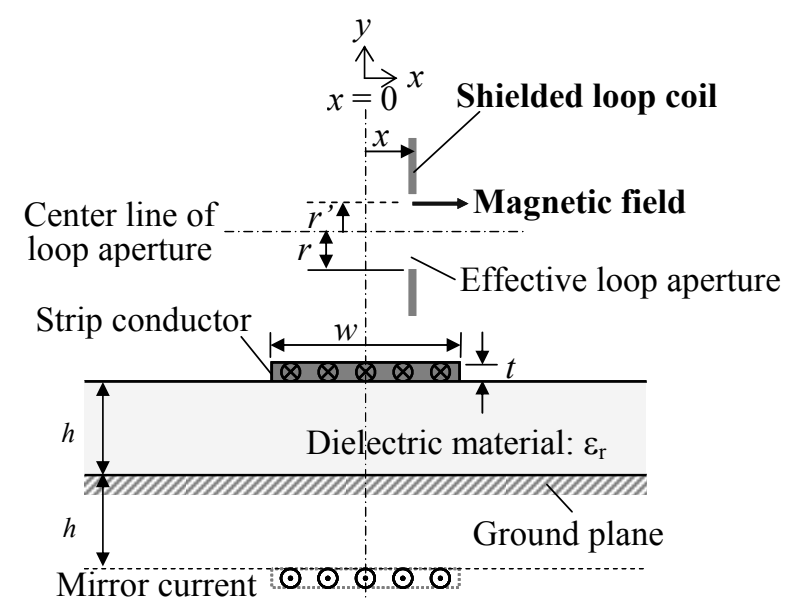

(b)

Fig. 3 Schematics of measurement setup. (a) Angled view. (b) Cross-sectional view. A microstrip line is used to evaluate basic characteristics of the new magnetic field probes. 
Table 2 Characteristics of microstrip lines.

\begin{tabular}{|c|c|c|c|}
\hline$w$ & $770 \mu \mathrm{m}$ & $60 \mu \mathrm{m}$ & $5 \mu \mathrm{m}$ \\
\hline$t$ & $35 \mu \mathrm{m}$ & $8 \mu \mathrm{m}$ & $0.2 \mu \mathrm{m}$ \\
\hline$h$ & $254 \mu \mathrm{m}$ & $50 \mu \mathrm{m}$ & $1 \mu \mathrm{m}$ \\
\hline$\varepsilon_{r}$ & 2.1 & 5.5 & 4.0 \\
\hline
\end{tabular}

relative dielectric constant of the dielectric in the microstrip line. We refer to the vertical distance from the upper edge of the strip conductor to the nearest edge of the shielded loop coil as the "lift-off." The minimum limit of the lift-off depends on the location accuracy of the scanning system, the flatness of the surface of the DUT, the distance from the coil edge to the probe edge, and so on. If a measurement point is fixed, the lift-off can be reduced to $1 \mu \mathrm{m}$. A microstrip line was connected to a signal generator or port 1 of a network analyzer from which power was input to the microstrip line. The probe was connected to a spectrum analyzer or port 2 of the same network analyzer. The probe was oriented as shown in Fig. 3 to detect the $x$-component of the magnetic field above the microstrip line. Three microstrip lines were used as a standard magnetic field generator. Table 2 lists their characteristics. The $770-\mu \mathrm{m}$-wide microstrip line has characteristic impedance of $50 \Omega$. The $770-\mu \mathrm{m}$ width is much larger than the dimensions of the new coil. The $60-\mu \mathrm{m}$-wide microstrip line was fabricated by using glass ceramic processes. The $60-\mu \mathrm{m}$ width is comparable to the typical width of global power lines on an LSI chip, whereas the dimensions of the microstrip line in the thickness direction are nearly or more than 10 times as large as those of a typical LSI chip. The 5 - $\mu \mathrm{m}$-wide microstrip line was fabricated by using thin-film processes. The 5- $\mu \mathrm{m}$ width is smaller than the typical width of global power lines on an LSI chip, whereas the dimensions of the microstrip line in the thickness direction are comparable to those of a typical LSI chip.

\subsection{Frequency characteristics}

The frequency-output characteristics of the probe were evaluated. In this evaluation, it was desirable that the electromagnetic field around the shielded loop coil be almost uniform, and the effect of disturbing the electromagnetic field caused by placing the coil be suppressed. To achieve this, a 770- $\mu$ m-wide microstrip line with characteristic impedance of $50 \Omega$ was used as the DUT, and the lift-off was maintained at $200 \mu \mathrm{m}$. Thus, both the lift-off and strip conductor width were larger than the loop size. The microstrip line was terminated by the $50-\Omega$ resister. The coil was placed at the center of the strip conductor, $x=0$. Figure 4 plots the frequency dependence of the ratio of the probe output voltages to the input voltages supplied to the microstrip line. The solid squares represent the data obtained up to $1 \mathrm{GHz}$ by using a spectrum analyzer and a signal generator. The open squares represent the data obtained above $1 \mathrm{GHz}$ by using a network analyzer. The measured data are proportional to the frequency up to about $7 \mathrm{GHz}$. When

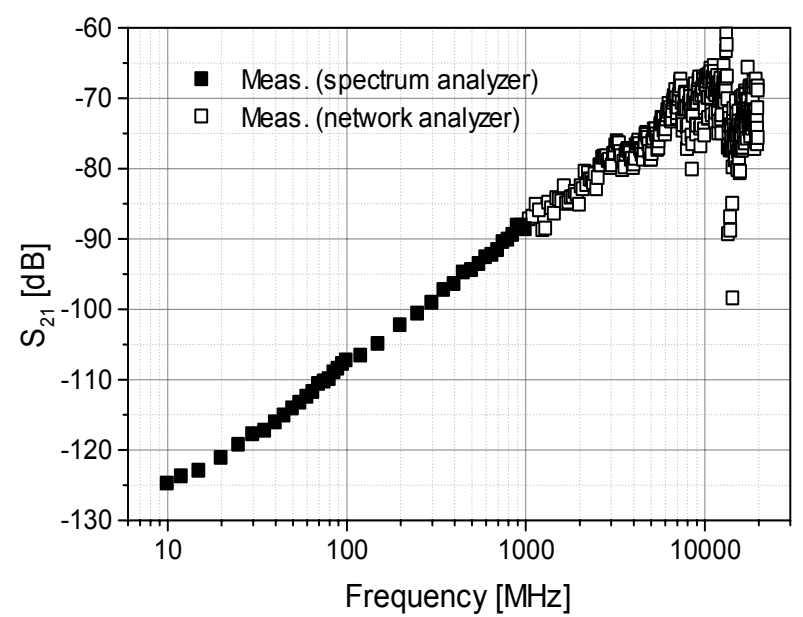

Fig. 4 Frequency characteristics of the new magnetic field probe. Measurements were taken above a 770 - $\mu \mathrm{m}$-wide microstrip line with $50-\Omega$ termination. Lift-off was maintained at $200 \mu \mathrm{m}$.

the frequency is larger than $7 \mathrm{GHz}$, peaks and dips due to resonance appear. This means that the probe output voltages are mainly induced by the rate of change in magnetic flux in the loop aperture up to about $7 \mathrm{GHz}$.

\subsection{Measurement of standing waves}

The probe's insensitivity to electric fields was evaluated by examining the probe output distribution in the $z$ direction over the $770-\mu \mathrm{m}$-wide microstrip line with a short termination at $z=0$. In this case, standing waves were formed over the microstrip line, and the distributions of the electric and magnetic fields are given by (1) and (2), respectively.

$$
\begin{aligned}
& |E(z)|=E_{\text {max }}|\sin (k z)| \\
& |H(z)|=H_{\text {max }}|\cos (k z)|
\end{aligned}
$$

Figure 3(a) shows the measurement setup. The coil was placed on the plane of $x=0$. The lift-off was maintained at $200 \mu \mathrm{m}$ for the same reason as described in the previous section. The measured distribution obtained with the new probe was compared with that obtained with our previous ceramic probe. The latter probe is already commercialized $^{3)}$. Figure 5 shows the measured probe output distributions and calculated electromagnetic field distributions in the $z$ direction at $1 \mathrm{GHz}$. These values are all normalized by their maximum values. In Fig. 5, $z$ represents the product of the electrical length and wavelength of the standing wave in the microstrip line. The solid squares and the open circles represent the measured results obtained with the new probe and with the previous ceramic probe. The solid and dash-dotted lines respectively represent the calculated magnetic and electric field distributions. The measured distributions are very close to the calculated magnetic field distribution. In Fig. 5, the minimum value obtained with our previous ceramic probe is nearly zero, whereas the minimum value 


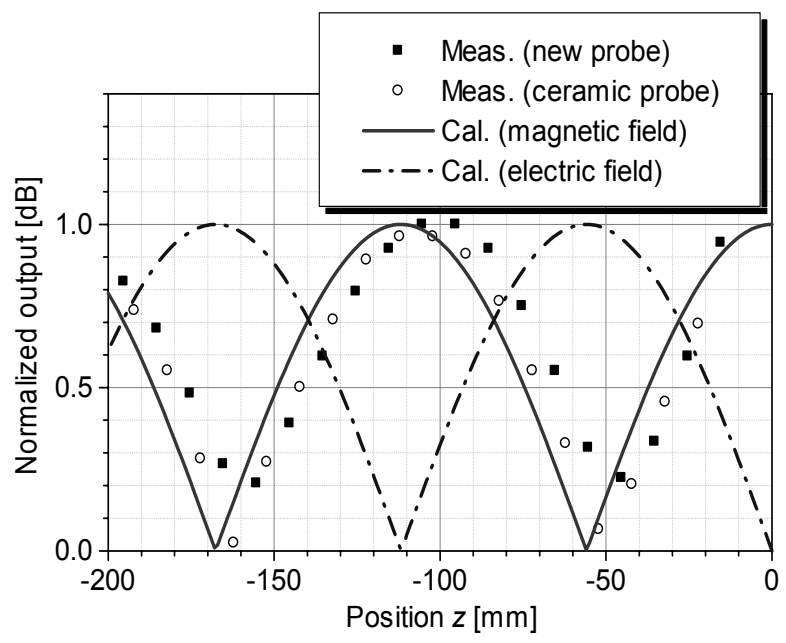

Fig. 5 Measured probe output distribution and calculated electromagnetic field distributions in the $z$ direction above the 770- $\mu \mathrm{m}$-wide microstrip line with a short termination at $z=0$, where standing waves were formed. Lift-off was maintained at $200 \mu \mathrm{m}$.

obtained with our new probe deviates from zero. This difference at the minimum points comes from the lower signal-to-noise ratio in the data obtained with the new probe. In Fig. 5, the measured probe output distributions obtained with our new and previous probes deviates from the calculated magnetic field distribution in the $\mathrm{z}$ direction. We consider that the difference between the measured distributions and the calculated magnetic field is due not only to the effect of the electric field but also to other factors, such as an error in compensating for the $z$-axis, a slight impedance mismatch at the connectors, etc. We plan to study the effect of the electric field on our new probe in the future. From these results, along with the frequency characteristics discussed above, we found that our new probes mainly detected the magnetic field.

\subsection{Measurement of spatial resolution}

There is no common definition of the probe's spatial resolution because it depends not only on the probe itself but also on the dimensions of the DUT. Thus, the spatial resolution must be evaluated with respect to each DUT. The spatial resolution of our new probe was evaluated by measuring the output distributions above the $60-\mu \mathrm{m}$-wide and 5- $\mu \mathrm{m}$-wide microstrip lines. In these measurements, each probe was scanned over the microstrip lines in the $x$ direction.

To demonstrate the higher spatial resolution of the new probe, the output distribution measured with the new probe was compared with that measured with our previous glass probe. The 60 - $\mu \mathrm{m}$-wide microstrip line was used as the DUT. The lift-offs were reduced closer to

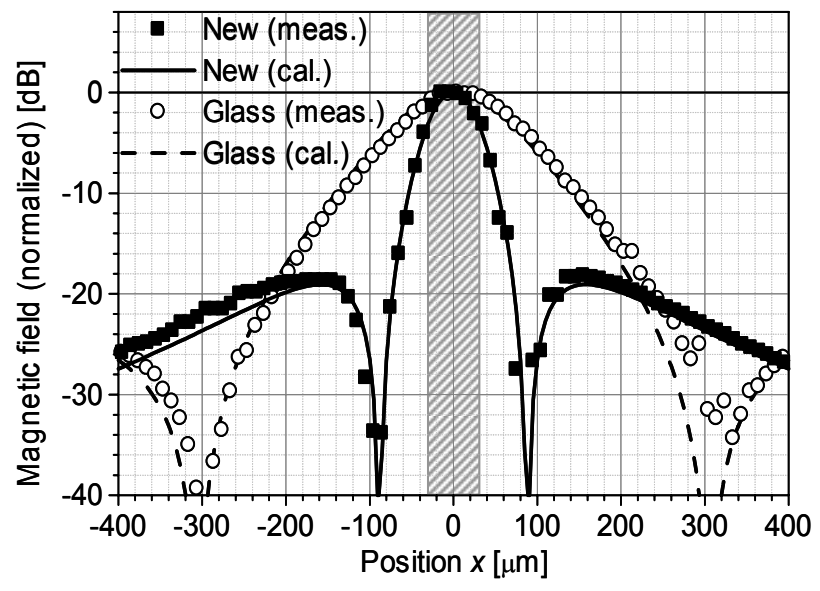

Fig. 6 Comparison of magnetic field distributions obtained with our new and previous probes along the $x$-axis over the 60 - $\mu \mathrm{m}$-wide microstrip line. In the evaluation with the new probe, the lift-off was maintained at $30 \mu \mathrm{m}$, whereas in the measurement with the previous glass probe the lift-off was maintained at $40 \mu \mathrm{m}$ with the previous glass probe.

the minimum limits. In the measurement with the new probe, the lift-off was maintained at $30 \mu \mathrm{m}$, whereas in the measurement with the previous glass probe the lift-off was maintained at $40 \mu \mathrm{m}$ with the previous glass probe. Figure 6 plots the measured and calculated magnetic field distributions obtained with the new and previous probes at $1 \mathrm{GHz}$. These distributions are all normalized by their maximum values. In calculation, the magnetic field distribution was obtained by averaging the $x$-component of the magnetic field in the loop; it is given by

$$
H_{\text {ave }}(x)=\frac{1}{2 r} \int_{-r}^{r} H_{x}\left(x, r^{\prime}\right) d r^{\prime}
$$

where $H_{x}\left(x, r^{\prime}\right)$ represents the $x$-component of the magnetic field at position $\left(x, r^{\prime}\right)$ generated by both the current in the strip conductor and its mirror current, as shown in Fig. 3(b). In this calculation, we assumed that the current density in the strip conductor was uniform. The solid squares and the solid line represent the measured and calculated distributions obtained with the new probe, while the open circles and the dash-dotted line represent the measured and calculated ones obtained with the previous glass probe, respectively.

In Fig. 6, both the measured results obtained with the new and previous probes are in good agreement with their calculated results. The measured distribution obtained with the new probe was much sharper than that obtained with the previous one. The distance from the origin of the $x$-axis to the point where it decreases by $6 \mathrm{~dB}$ can be regarded as an index for evaluating the spatial resolution of these probes. The $6-\mathrm{dB}$ decrease point of the new probe was $40 \mu \mathrm{m}$, which is less than half that of the previous glass probe $(100 \mu \mathrm{m})$. This result demonstrates the higher 


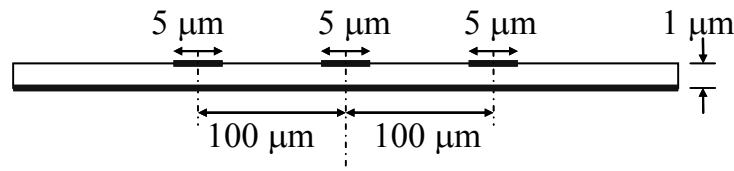

(a)

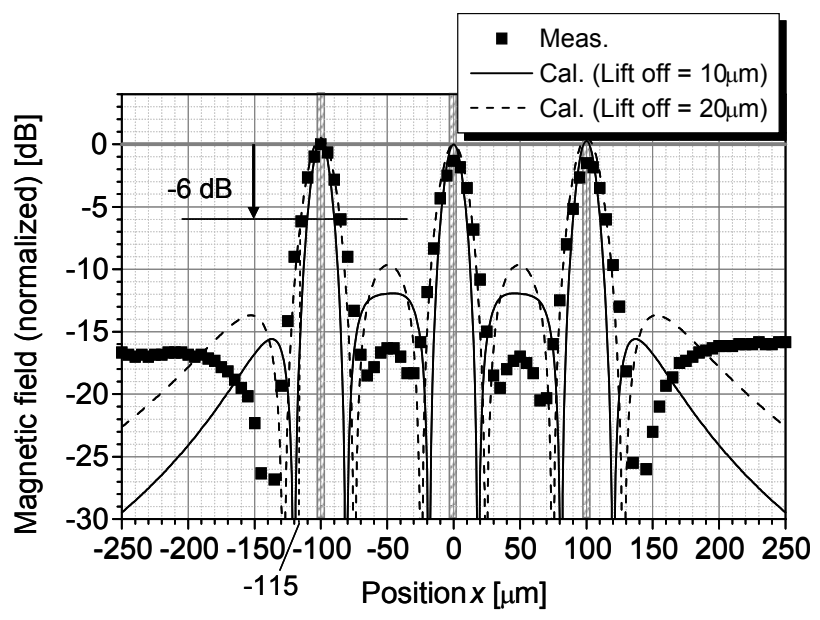

(b)

Fig. 7 (a) Cross-sectional view of the DUT with 5 - $\mu \mathrm{m}$-wide lines. Line pitch is $100 \mu \mathrm{m}$. (b) Measured and calculated magnetic field distributions obtained with the new probe over the DUT. Lift-off was maintained at $10 \mu \mathrm{m}$.

spatial resolution of the new probe.

The magnetic field distribution was also measured over the DUT with the 5- $\mu \mathrm{m}$-wide lines. Figure 7(a) shows the structure of the DUT. The DUT has three strip conductors and the ground plane. The line pitch is $100 \mu \mathrm{m}$. When the new probe is scanned over the DUT (or an LSI chip) using our scanning system, the lift-off can be reduced to about $10 \mu \mathrm{m}$. In this measurement, the lift-off was maintained at $10 \mu \mathrm{m}$. Figure 7(b) shows the measured and calculated magnetic field distributions obtained with the new probe at $1 \mathrm{GHz}$. The solid squares represent the measured distribution obtained, while the solid line and the dash-dotted line represent the calculated ones when the lift-off was maintained at $10 \mu \mathrm{m}$ and $20 \mu \mathrm{m}$, respectively. All data are normalized by their maximum values. In the measurement, three sharp peaks were obtained over the strip conductors. The distance from the maximum point to the $6-\mathrm{dB}$ decrease point was reduced to only $15 \mu \mathrm{m}$. This result means that the new probe achieved $10-\mu \mathrm{m}$-class high spatial resolution. In Fig. 7(b), the measured data are in good agreement with the dash-dotted line rather than with the solid line. This is due to a positioning error in the $y$ direction.

\subsection{Estimation of spatial resolution at the LSI chip level}

We assume that the new probe has spatial resolution high enough to obtain the absolute value of current flowing on each strip line if the new probe separately detects magnetic field generated from each power line. Therefore, we did some estimations to determine which DUT configuration would enable the new probe to separately detect the magnetic field generated from each power line.

In this estimation, calculations based on Eq. (3) were done. Figures 6 and 7(b) show that our calculations are

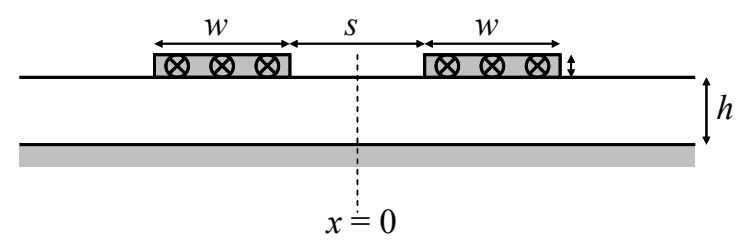

(a)

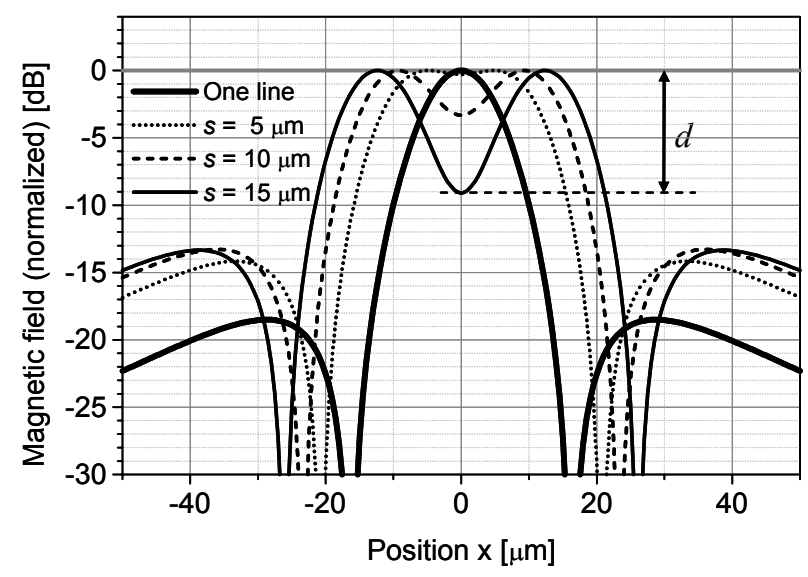

(b)

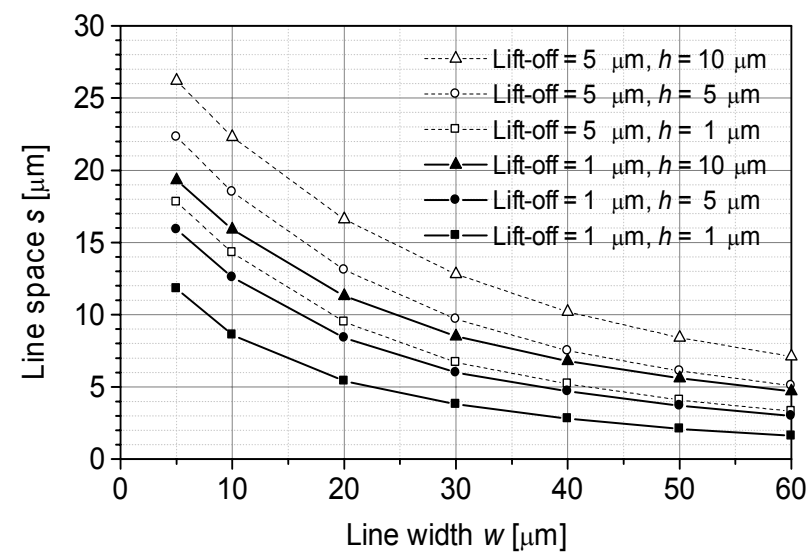

(c)

Fig. 8 (a) Cross-sectional view of the DUT. (b) Calculated magnetic field distributions obtained with new probe over the DUT. Lift-off was maintained at 1 $\mu \mathrm{m}$. (c) Relationship between dimensions $w$ and $s$ when the depth of dip $d$ is larger than $6 \mathrm{~dB}$. 
reliable. The structure of lines on an LSI chip was modeled by using the DUT shown in Fig. 8(a). The DUT has two strip conductors as well as the ground plane. In Fig. 8(a), the dimensions $h, w$, and $s$ represent the thickness of the dielectric, the width of each strip conductor, and the distance between the two strip conductors, respectively. The origin of the $x$-axis was set at the center of the gap between the two strip conductors. The currents flowing through the two strip conductors are assumed to have the same phase and value. In terms of spatial resolution, this configuration is much tighter than when the currents flowing through the two strip conductors have reversed phases, because the former condition needs a much larger dimension $s$ than the latter one to separately detect the magnetic field generated from each power line.

Figure 8(b) shows the calculated magnetic field distributions obtained with the new probe over the DUT. In this calculation, the dimensions $w, t$, and $h$ were set at $10,0.1$, and $5 \mu \mathrm{m}$, respectively. These values are representative of global power lines on an LSI chip. The lift-off was maintained at $1 \mu \mathrm{m}$. In Fig. 8(b), the dotted line, dashed line, and solid line represent the calculated distributions when dimension $s$ was set at 5, 10, and 15 $\mu \mathrm{m}$, respectively. The bold line represents the calculated distributions when there is only one strip conductor, and the center of the strip conductor is set at $x=0$. All data are normalized by their maximum values. When $s=5 \mu \mathrm{m}$, a dip is hardly observed at $x=0$. When $s=10 \mu \mathrm{m}$, a dip is observed at $x=0$. The depth of $\operatorname{dip} d$ is $3.3 \mathrm{~dB}$. When $s=$ $15 \mu \mathrm{m}$, depth $d$ is $9.1 \mathrm{~dB}$. These results show that the larger the dimension $s$ is, the more sharply the two peaks of the magnetic field distribution around the two strip conductors are separated, with depth $d$ getting deeper. Therefore, depth $d$ can be regarded as an index of separation of the magnetic field distribution around the two strip conductors. Considering the performance of the measurement equipment, depth $d$ should be larger than some value in order to detect the dip distinctly. Therefore, we presume that the new probe separately detected the magnetic field generated from each power line if depth $d$ is larger than $6 \mathrm{~dB}$.

Finally, we did some estimations to determine the configuration that would enable depth $d$ to be more than 6 $\mathrm{dB}$, when the magnetic field distribution is obtained with the new probe over the DUT shown in Fig. 8(a). Figure 8(c) presents the relationship between the dimensions $w$ and $s$ that satisfy this condition. The smaller dimension $h$ is, the smaller dimension $s$ can be because the effect of the mirror current is large when dimension $h$ is small.
If a power line is traced next to a ground line, currents in the power and ground lines usually flow in the opposite direction. In this case, there is a strong possibility that the new probe separately detects magnetic field generated from each power line even if the distance between the two neighboring lines is less than dimension $s$, as shown in Fig. 8(c). Our challenge in the future is to evaluate the effect of Si substrates with larger resistivity on the probe's spatial resolution.

\section{Conclusion}

In this study, a shielded loop coil was miniaturized in order to enhance the probe's spatial resolution. The minimum outer size of the new coil was $50 \times 22 \mu \mathrm{m}$. The experiment demonstrated that the new probe has a much higher spatial resolution than our previous glass probe. In measurements done with the new probe above 5 - $\mu$ m-wide microstrip lines, we found that the new probe achieved $10-\mu \mathrm{m}$-class spatial resolution. We estimated the configuration of the lines on an LSI chip that would enable the new probe to achieve a spatial resolution high enough to obtain the absolute value of high-frequency power currents on LSI chips. This new probe is expected to be used to search for high-frequency noise sources by tracing the paths of strong high-frequency currents on LSI chips, to directly verify a circuit design to suppress EMI, to verify simulation models of LSI power circuits, and other such applications.

\section{References}

1) T. Sudo, H. Sasaki, N. Masuda, and J. L. Drewniak: IEEE Trans. Adv. Packag., 27, 304 (2004).

2) M. Iwanami, S. Hoshino, M. Kishi, and M. Tsuchiya: IEEE Symp. EMC, 1, Boston, (2003), p. 347.

3) N. Masuda, N. Tamaki, T. Watanabe, and K. Ishizuka: NEC Res. \&Develop., 42, 246 (2001).

4) N. Masuda, N. Tamaki, T. Kuriyama, J. C. Bu, M. Yamaguchi, and K. I. Arai: IEEE Symp. EMC, 1, Boston, (2003), p. 80.

5) N. Tamaki, N. Masuda, T. Kuriyama, J. C. Bu, M. Yamaguchi, and K. I. Arai: Electronics and Communications in Japan, Part2, 88, 37(2005).

6) M. Yamaguchi, S. Yabukami, and K. I. Arai: IEEE Trans. Magn., 32, 4941 (1996).

7) N. Ando, N. Masuda, N. Tamaki, T. Kuriyama, S. Saito, S. Kato, K. Ohashi, M. Saito, and M. Yamaguchi: IEEE Int. Symp. EMC, vol. 2, Santa Clara, (2004), p. 357.

8) Y. Nonaka, H. Honjo, T. Toba, S. Saito, T. Ishi, M. Saito, N. Ishiwata, and K. Ohashi: IEEE Trans. Magn., 36, 2514 (2000).

Received May 24, 2005; Accepted Apr. 14, 2006. 